\title{
$4 \mathrm{G}$ 室分向 $5 \mathrm{G}$ 演进建设方案浅谈
}

\author{
胡文星朱呍滋周博明张虹李罡 \\ 中国移动通信集团设计院有限公司湖南分院，湖南长沙 410003
}

[摘要] 当前通信行业正处于 $4 G$ 向 $5 G$ 演进的关键时期, 文章主要探讨如何在室内分布系统建设中实现低成本建网, 既充分利 用现有 $4 G$ 网络资源, 又发挥 $5 G$ 高容量优势。分析了传统 DAS 和数字化室分中具有代表性的两种建设方式, 分别从不同维度 分析了同轴电缆系统和分布式皮基站向 $5 \mathrm{G}$ 演进方式, 并给出网络改造的经验。针对同轴电缆系统, 分别从空间传播链路、器 件支持能力、施工难度三方面, 对 $4 \mathrm{G}$ 升级改造可行性进行了探讨; 针对分布式皮基站, 分别从覆盖能力、施工难度、系统维 护、容量规划以及功率配置五方面, 分析了其向 $5 G$ 演进的优势。

[关键词] $5 \mathrm{G}$; 室内分布; 传统 DAS; 数字化室分

DOI：10.33142/sca.v3i4.2186 中图分类号：TN929.5 文献标识码：A

\section{Brief Discussion on the Evolution of 4G Room to 5G}

HU Wenxing, ZHU Xuanzi, ZHOU Boming, ZHANG Hong, LI Gang

China Mobile Group Design Institute Co., Ltd. Hunan Branch, Changsha, Hunan, 410003, China

\begin{abstract}
At present, the communication industry is in a critical period of evolution from 4G to 5G. This paper mainly discusses how to build a low-cost network in the construction of indoor distribution system, which not only makes full use of the existing $4 \mathrm{G}$ network resources, but also gives full play to the advantages of 5G high-capacity. This paper analyzes two typical construction methods of traditional DAS and digital room, analyzes the evolution mode of coaxial cable system and distributed Pico base station to 5G from different dimensions, and gives the experience of network transformation. For the coaxial cable system, the feasibility of $4 \mathrm{G}$ upgrading is discussed from three aspects: space transmission link, device support ability and construction difficulty; for distributed Pico base station, the advantages of its evolution to $5 \mathrm{G}$ are analyzed from five aspects of coverage capacity, construction difficulty, system maintenance, capacity planning and power configuration.
\end{abstract}

Keywords: 5G; indoor distribution; traditional DAS; digital indoor distribution

\section{引言}

统计表明, 4G 时代有 80\%的业务发生在室内, 4G 室分建设着力于中大型居住群, 商用建筑、大型场馆、交通枢纽 及高校、隧道地铁等场景, 根据各个场景的特点, 分别采用了同轴电缆系统、分布式皮站、光纤分布系统、一体化皮 站等不同的覆盖方式, 其中, 以同轴电缆系统和分布式皮站为主, 占比约 $90 \%$ 。在未来的第五代移动通信（the 5 th generation wireless systems, 5G）时代, 室内场景依然是移动数据业务的主力区域。但 $5 G$ 网络受频段高, 新增站 址困难、单站成本更高、早期投资回报率较低等因素影响, 为此, 如何既发挥 $5 \mathrm{G}$ 的高容量优势, 同时充分利用现有 $4 \mathrm{G}$ 网络投资, 进而实现低成本建网, 成为了室内分布系统建设的热点话题。

\section{$15 G$ 室分演进可行性分析}

2017 年 11 月 10 日, 工信部正式发布《工业和信息化部关于第五代移动通信系统使用 $3300-3600 \mathrm{MHz}$ 和 $4800-$ $5000 \mathrm{MHz}$ 频段相关事宜的通知》, 明确表示 3300-3400MHz 频段原则上限室内使用。本文将结合中国移动室分网络现状, 对 5G 室分建设进行分析。目前, 中国移动获得了 2.6G、4.9G 频段的 $5 \mathrm{G}$ 试验频率资源, 相对于原有的 $4 \mathrm{G}$ 室分频段 $2.3 \mathrm{G}$, 需从多维度全面分析建设方案的可行性。

室内分布系统根据传输媒介分为射频无源分布系统、射频有源分布系统、光纤分布方式及泄漏电缆分布方式, 目 前室内覆盖方式主要有两种: 无源分布系统和有源分布系统。无源分布系统主要为传统的同轴电缆系统, 有源分布系 统主要为数字化的分布式皮基站。在 $4 \mathrm{G}$ 网络时代, 无源分布系统凭借其低廉的建设成本, 在话务量需求不高的场景能 够和有源分布系统抗衡。但在 $5 \mathrm{G}$ 时代, 在大带宽、高速率、高频段面前, 无源分布系统已无法跟上时代进步的步伐。 
从技术可行性上看, 面向 $5 \mathrm{G}$, 传统无源分布系统已经无法满足其速度和容量的需求, 数字化的有源分布系统是必由之 路。面向 $5 \mathrm{G}$ 演进, 数字化有源分布系统涉及改造最少, 能有效保障工程可实施性。在 $5 \mathrm{G}$ 发牌后, 能否快速叠加 $5 \mathrm{G}$ 网 络、抢占 $5 \mathrm{G}$ 先发优势, 现有的 $4 \mathrm{G}$ 室分系统通过何种方式向 $5 \mathrm{G}$ 室分系统演进, 满足 $5 \mathrm{G}$ 网络的覆盖要求, 这些都是目 前运营商需要提前考虑的问题。

ITU-R 定义的 $5 \mathrm{G}$ 关键能力指标如表 1 所示:

表 $15 \mathrm{G}$ 移动通信关键性能指标

\begin{tabular}{c|c}
\hline 关键能力 & 指标 \\
\hline 用户体验 & DL $>100 \mathrm{Mbps}, \mathrm{UL}>10 \mathrm{Mbps}$ \\
\hline 区域流量 (到 2020 年) & $2.5 \mathrm{Mbps} / \mathrm{m}^{2}$ (室内高密场景), $0.5 \mathrm{Mbps} / \mathrm{m}^{2}$ (室内普通场景) \\
\hline 频谱效率 & 3 倍 \\
\hline 时延 & $1 \mathrm{~ms}$ \\
\hline 连接定位精度 & 1 米 \\
\hline 网络能效 & 100 倍 \\
\hline 峰值速率 & $10 \mathrm{Gbps}$ \\
\hline 连接密度 & $1 \mathrm{M} \mathrm{devices} / \mathrm{km}^{2}$ \\
\hline 网络可靠性 & 0.99999 \\
\hline 移动性 & $10 \mathrm{~km} / \mathrm{h}$ \\
\hline
\end{tabular}

为实现上述各性能指标， $5 \mathrm{G}$ 的关键技术包括：C-band 100MHz、4T4R、多频多模协同、弹性扩容、高精度定位、精 准切片、精准头端管理、精准能效管理等。在 $5 \mathrm{G}$ 的无线接口中, 4T4R 是基本要求, 高阶的 MIM0 (多入多出技术) 是 5G 网络提升速率的基本手段, 即使室内覆盖也必然是 4T4R 的 MIM0 起步。

目前各大运营商已规划建设的 $4 \mathrm{G}$ 有源分布系统如何平稳的过渡演进到 $5 \mathrm{G}$ 室分系统, 仍然需要做大量的准备工作。 现有 $4 \mathrm{G}$ 有源分布系统在改造时如何做到工作量最少、施工难度最低, 以及新建的有源分布系统如何提前布局, 值得我 们进一步探讨。

\section{2 演进改造方案}

\section{1 同轴电缆系统改造}

同轴电缆系统的改造, 在 $4 G$ 室分向 $5 G$ 演进中具有重要的现实意义。以中国移动为例, 同行业相比, 其 $4 G$ 室分建 设具有较为突出优势; 但在存量 $4 G$ 室分基站中, 传统同轴电缆系统占比较高, 已逾九成。因此 $5 G$ 建设阶段, 如何充 分发挥室内覆盖存量优势, 确保室内覆盖性能, 保障网络竞争优势, 显得尤为重要, 针对传统同轴电缆的升级改造势 在必行。

以下将从链路预算、器件支持能力、施工难度等几个方面分析传统室分同轴电缆系统 $4 \mathrm{G}$ 向 $5 \mathrm{G}$ 演进的可行性。 在空间传播链路预算方面，室内覆盖电波传播模型可由下式描述:

PL $(d, f)=38.4+2010 g(d)+20 \log (f / 2000)+\beta \times d+F A F \quad(d B)$

式中: $d$ 一一单位: $m ; f$ 一一单位: $\mathrm{MHz} ; \beta$ 一一路径损耗因子 $(0 \sim 1.6 \mathrm{~dB} / \mathrm{m}$, 典型取 $0.5 \mathrm{~dB} / \mathrm{m}) ; \mathrm{FAF}$ 一一穿墙损耗。

38. 900 协议针对 Indoor 2 Indoor office NLOS 场景模型

PLInH-NLOS $=38.3 \log 10(\mathrm{~d})+17.30+24.9 \log 10(\mathrm{f})+\mathrm{FAF}$

式中: d-—单位: m; f一一单位: GHz; FAF一一穿墙损耗。 
链路预算结果如表 2 所示:

表 2 不同模型下的传播损耗链路预算结果

\begin{tabular}{|c|c|c|c|c|c|c|c|c|c|}
\hline 覆盖距离 (m) & 模型 & $1850 \mathrm{M}$ & $2150 \mathrm{M}$ & $2350 \mathrm{M}$ & $2650 \mathrm{M}$ & $3450 \mathrm{M}$ & $3700 \mathrm{M}$ & $4450 \mathrm{M}$ & $4900 \mathrm{M}$ \\
\hline \multirow{2}{*}{10} & 模型一 & 70.7 & 72.0 & 72.8 & 73.8 & 79.1 & 79.7 & 82.3 & 83.2 \\
\hline & 模型二 & 70.3 & 71.9 & 72.8 & 74.1 & 80.0 & 80.7 & 83.7 & 84.8 \\
\hline \multirow{2}{*}{15} & 模型一 & 76.7 & 78.0 & 78.8 & 79.9 & 85.2 & 85.8 & 88.4 & 89.2 \\
\hline & 模型二 & 77. 0 & 78.6 & 79.6 & 80.9 & 86.7 & 87.5 & 90.5 & 91.5 \\
\hline \multirow{2}{*}{20} & 模型一 & 81.7 & 83.0 & 83.8 & 84.9 & 90.2 & 90.8 & 93.4 & 94.2 \\
\hline & 模型二 & 81.8 & 83.4 & 84.4 & 85.7 & 91.5 & 92.3 & 95.3 & 96.3 \\
\hline \multirow{2}{*}{25} & 模型一 & 86.2 & 87.5 & 88.3 & 89.3 & 94.6 & 95.2 & 97.8 & 98.6 \\
\hline & 模型二 & 85.5 & 87.1 & 88.1 & 89.4 & 95.2 & 96.0 & 99.0 & 100.0 \\
\hline
\end{tabular}

注: 不同材质穿墙损耗差异较大, 本文中按相对于 $2 \mathrm{GHz}$ 频段, $3 \mathrm{GHz}$ 频段穿损高 $3 \mathrm{db}, 4 \mathrm{GHz}$ 频段高 $4 \mathrm{db}$ 考虑。

由式 (1)、（2）以及表 2 分析可知，相对于 $2.3 \mathrm{G}$ 频段， $2.6 \mathrm{G}$ 频段传播损耗约增加 $1 \mathrm{db}, 4.9 \mathrm{G}$ 空间传播损耗约增加 $10 \mathrm{db}$ 。如使用 $2.6 \mathrm{G}$ 频段进行室分覆盖, 基本在保持现有天线出口功率的情况下, 可以满足覆盖需求。但如使用 $4.9 \mathrm{G}$ 频段进行室分覆盖，需加大天线入口功率 10db 以上。由此可见，随着频段的增加入口功率也随之增加。

其次, 在器件支持能力方面, 目前已有部分厂商推出支持至 $6 \mathrm{GHz}$ 产品, 可对现有 $4 \mathrm{G}$ 天线进行替换; 而无源器件 方面, 市场中仅存在最高 $3.6 \mathrm{GHz}$ 的相关产品, 如使用 $4.9 \mathrm{GHz}$ 进行室内覆盖, 则目前暂无可替换产品; 在馈线方面, 市场中仅存在最高 $3 \mathrm{GHz}$ 产品, 如使用 $4.9 \mathrm{GHz}$ 进行室内覆盖, 同样暂无可替换产品。且随着频率的增高, 馈线的损耗 越来越大, 需要更高的信源输出功率, 如表 3 所示。

表 3 不同频段下的馈线损耗

\begin{tabular}{c|c|c|c|c|c|c|c}
\hline 每百米损耗 $(\mathrm{dB} / 100 \mathrm{~m})$ & $900 \mathrm{M}$ & $1800 \mathrm{M}$ & $2100 \mathrm{M}$ & $2300 \mathrm{M}$ & $2600 \mathrm{M}$ & $3500 \mathrm{M}$ & $4900 \mathrm{M}$ \\
\hline $7 / 8^{\prime \prime}$ & 3.8 & 5.5 & 6.1 & 6.4 & 6.8 & 8 & 9.8 \\
\hline $1 / 2^{\prime \prime}$ & 7.1 & 10.3 & 11.2 & 11.8 & 12.6 & 15 & 18 \\
\hline
\end{tabular}

目前现网 $4 \mathrm{G}$ 边缘场强为 $-105 \mathrm{dBm}$, 假设 $5 \mathrm{G}$ 边缘场强要求为 $-110 \mathrm{dBm}$, 则边缘场强差异约 $5 \mathrm{~dB}$ 。如使用 $2.6 \mathrm{G}$ 频段进 行室分覆盖, 基本可以做到同点位替换信源; 如使用 $4.9 \mathrm{G}$ 频段进行室分覆盖, 在不增加天线点位且同点覆盖前提下, 需要增加大量的 $5 \mathrm{G}$ 信源设备或极大的提升 $5 \mathrm{G}$ 信源发射功率, 然而在工程实施中较难实现。

再次, 在施工难度方面, 现有 $4 \mathrm{G}$ 同轴电缆室分为 $1 \mathrm{~T} 1 \mathrm{R}$ 或 $2 \mathrm{~T} 2 \mathrm{R}, 5 \mathrm{G}$ 室分要求 $4 \mathrm{~T} 4 \mathrm{R}$, 在建设过程中存在着以下几个 方面的问题:

(1) 需要安装 RRU, 无安装空间, 无法接电, 传输配套困难;

（2）改造双路或 4 路 DAS 需要新建天馈，天花板和弱电井可能无法安装，施工难度增加四倍;

(3) 天线和无源器件需要更换, 物业不允许大规模动天花板;

(4) 无法找到需替换的器件。

通过对链路预算、器件支持能力、施工难度三方面的分析, 同轴电缆系统进行 $5 \mathrm{G}$ 改造难度较大, 在工程实施过程 中较难实现。 


\section{2 分布式皮站改造}

数字化室分是未来的发展趋势, 分布式皮站是目前最主要的数字化室分方式。近年来, 数字化室分比例逐步提高, 对现网分布式皮站的改造需同步实施。分布式皮站向 $5 \mathrm{G}$ 演进有两种方式: 一种采用 $5 \mathrm{G}$ 单模设备, 如图 1 所示, BBU 增加 $5 \mathrm{G}$ 基带板, 叠加一个支持 $5 \mathrm{G}$ 的 rHUB, 新增一根 CAT $6 \mathrm{~A}$ 网线并增加 $5 \mathrm{G} \mathrm{pRRU;} \mathrm{由于} 5 \mathrm{G}$ 要求峰值速率达到 $10 \mathrm{Gbps}$, 因此网线必须使用超六类线; 另外一种即采用支持 $5 \mathrm{G}$ 的多模设备, 如图 2 所示, BBU 增加 $5 \mathrm{G}$ 基带板，拆除原有 $r H U B 、$

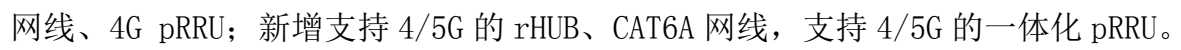

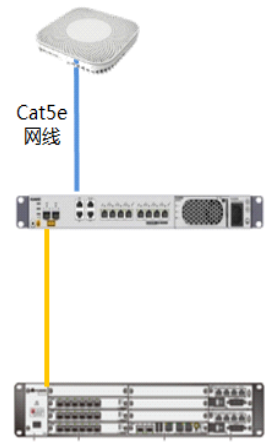

图 1 分布式皮站向 $5 G$ 演进（单模设备方式）
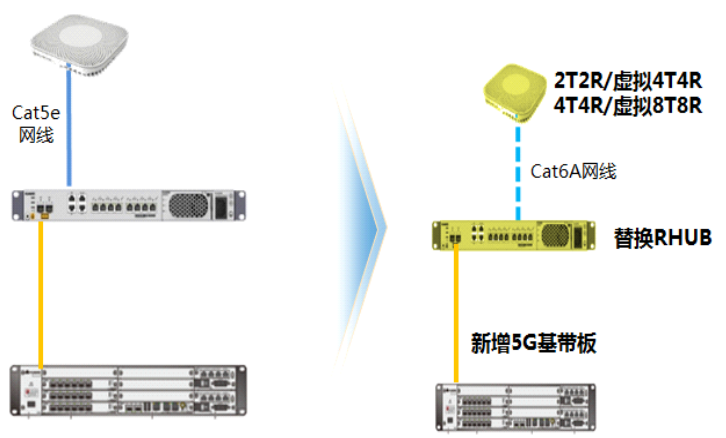

图 2 分布式皮站向 $5 G$ 演进（多模设备方式）

以下将分别从覆盖能力、施工难度、容量规划以及功率配置三方面进行分析。

(1) 覆盖能力

分布式皮站向 $5 \mathrm{G}$ 演进的优势显著, 首先可通过增加 pRRU 功率使 4T4R 实现与原 $\mathrm{pRRU}$ 相同的覆盖能力, 根据某厂 家仿真结果如表 4 所示, 目前典型场景实际工程部署 pRRU 间距为 30 米左右, 在现有分布式皮站点上替换 $5 \mathrm{G}$ 设备即可。

表 4 某厂家 PRRU 覆盖能力仿真结果

\begin{tabular}{c|c|c}
\hline 方案对比 & $4 G 2 \mathrm{~T} 2 \mathrm{R}$ & $5 \mathrm{G} 4 \mathrm{~T} 4 \mathrm{R}$ \\
\hline 带宽 & $20 \mathrm{MHz}$ & $100 \mathrm{MHz}$ \\
\hline 覆盖半径 & $29.2 \mathrm{~m}$ & $30.8 \mathrm{~m}$ \\
\hline $\mathrm{pRRU}$ 间距 & $58.4 \mathrm{~m}$ & 61.6 \\
\hline
\end{tabular}

（2）施工难度

分布式皮站能降低馈缆施工难度，目前各设备厂商均支持或计划支持 $5 \mathrm{G}$ 分布式皮站，但均需超六类线连接，如图 3 所示。现网的分布式皮站点大多使用五类线，需对此部分进行改造，但相对于传统室分需布放 4 路馈线，难度大大降低。

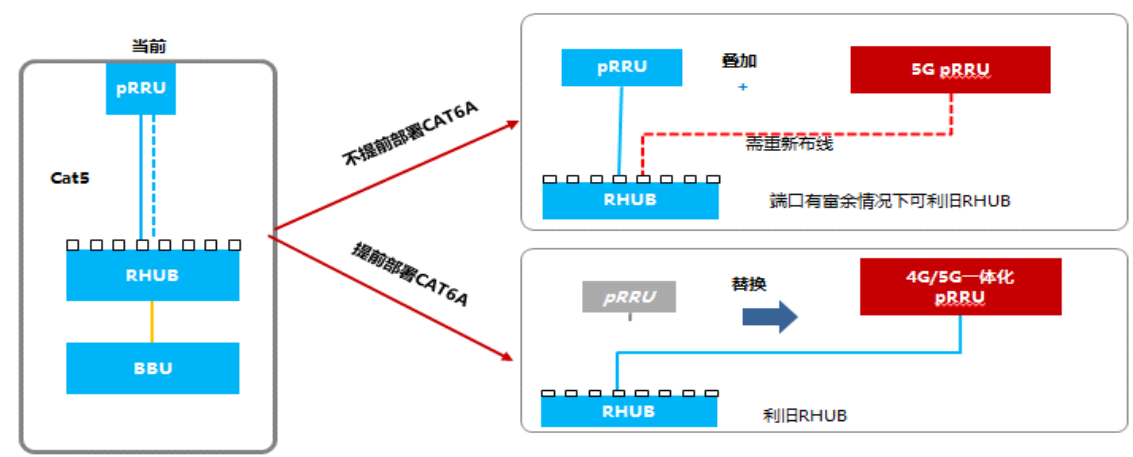

图 3 分布式皮站向 $5 \mathrm{G}$ 演进施工示意图 
（3）容量规划及功率配置

分布式皮站可实现故障点位及时可视、实时 pRRU 级业务可视, 以及功率配置灵活, 易于小区分列和小区合并, 软 件实现容量扩容。可随时了解设备实际部署位置, 精准楼层位置信息, 指导高效排障, 同时保证高效运维, 从宏观到 微观 (楼宇-楼层-pRRU) 实时监控。pRRU 级覆盖可视能远程发现弱覆盖, 并及时做出方案调整, pRRU 级干扰可视可远 程精确识别干扰, 并快速消除。

综上所述, 面向 $5 G$ 演进分布式皮站所进行改造的难度大大小于同轴电缆系统。若使用 $2.6 \mathrm{G}$ 频段进行室分覆盖, 基本可以实现现网 $4 \mathrm{G}$ 同轴电缆站点单路和双路 $5 \mathrm{G}$ 升级, 但 $4 \mathrm{~T} 4 \mathrm{R}$ 基本无法实现。若如使用 $4.9 \mathrm{G}$ 频段进行室分覆盖, 需新增大量的 $5 \mathrm{G}$ 信源, 且现有无源器件及馈线暂不支持, 无法实现现网 $4 \mathrm{G}$ 同轴电缆升级至 $5 \mathrm{G}$ 。分布式皮站可较好的 满足 $5 G$ 建设需求。

\section{3 改造投资对比}

根据链路预算及器件支持能力情况, 针对同轴电缆系统和分布式皮基站覆盖方式, 选取了高校和医院 2 个代表性 场景, 分新建和改造两种情况进行不同频段的投资测算分析。如图 4 所示，为两种覆盖方式的改造思路:

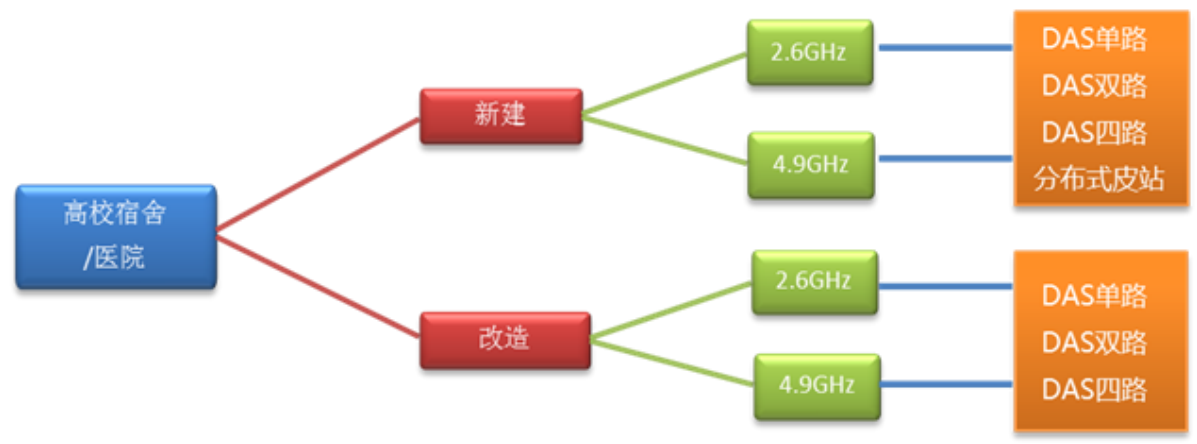

图 4 两种场景下 4G 向 5G 改造思路概览

根据以上改造方式，如图 5 和图 6 所示，可分别计算在 $2.6 \mathrm{G}$ 频段及 $4.9 \mathrm{G}$ 频段的改造投资情况。

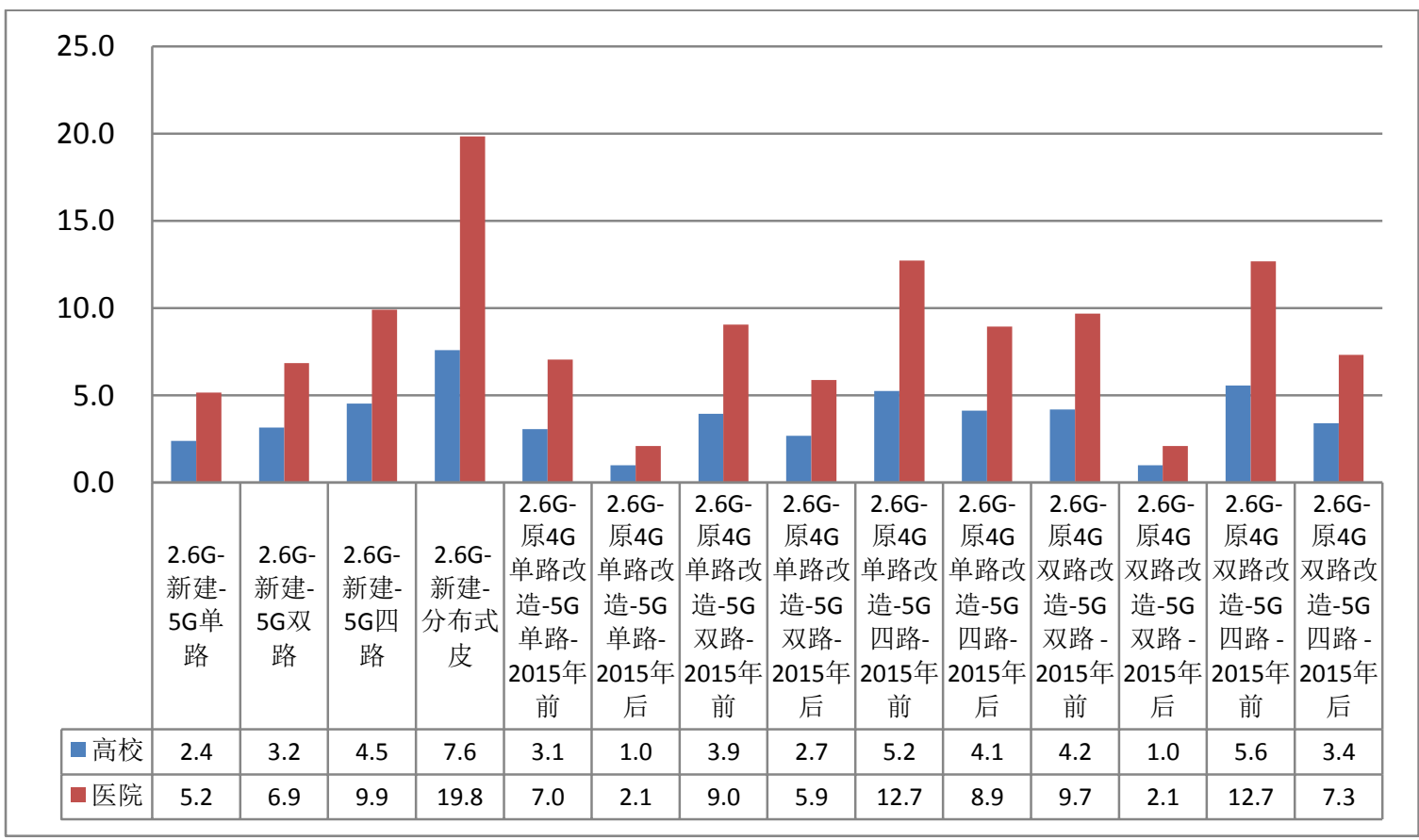

图 5 两种典型场景下 2. 6G 频段的改造投资对比 


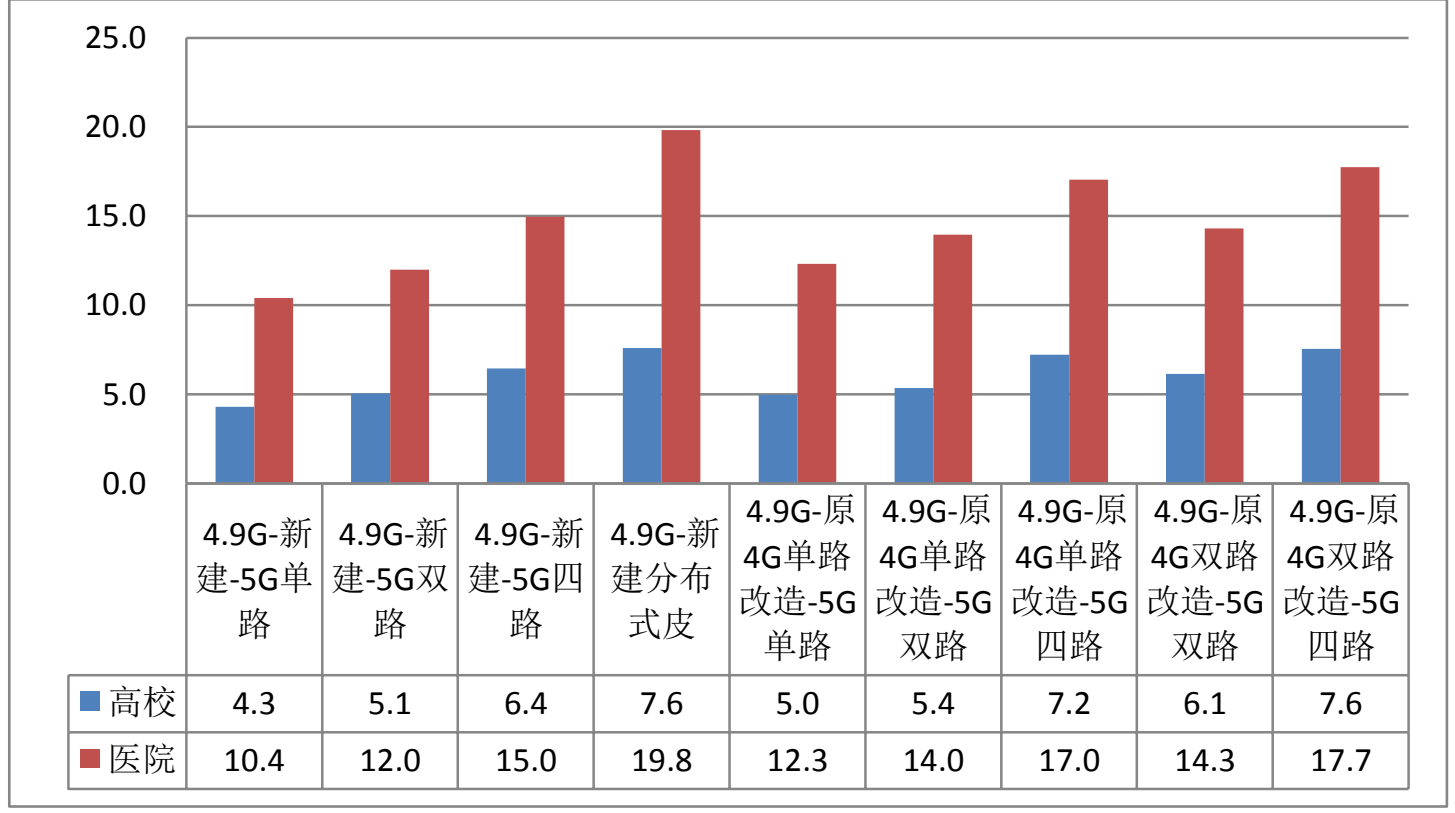

图 6 两种典型场景下 4.9G 频段的改造投资对比

通过上述分析, 不难判断未来 $5 \mathrm{G}$ 分布式皮设备成本相对于 $4 \mathrm{G}$ 预计增加 $80 \%$, $5 \mathrm{G}$ 信源相对于 $4 \mathrm{G}$ 信源设备成本预计 增加 1.5 倍以上; 因此无论何种频段, 新建/改造分布式皮站的投资仍远高于传统室分新建/改造投资。

\section{3 结语}

针对未来室分建设方案问题, 应优先考虑方案的可实施性和投资回报比, 建议高价值, 高流量空旷场景, 如大型 场馆, 会展中心, 交通枢纽, 机场等优选分布式皮站, 并提前部署 CATA6 网线, 方便后继 $5 \mathrm{G}$ 演进; 对现网存量的同轴 电缆系统站点，应优选高价值场景进行 $5 G$ 改造，并待 $5 G$ 产品技术参数及价格确定后，进行投资分析，因地制宜的选 择改造现有 $4 \mathrm{G}$ 同轴电缆系统或同址新建 $5 \mathrm{G}$ 分布式皮站。

\section{[参考文献 $]$}

[1]陈崴嵬,刘光海,杨安琪, 等. $5 \mathrm{G}$ 通信建设工程技术前沿报告 $[\mathrm{S}]$.中国通信学会, 2018.

[2]洪康. 5G 网络室内覆盖解决方案的分析 [J]. 信息通信, 2017,8(1):259-260.

[3] 黄海晖,刘大洋. 5G 时代室内覆盖解决方案综述 $[J]$. 移动通信, 2019, 43(6) : 42-46.

作者简介: 胡文星 (1983-), 男, 毕业院校于济南大学, 所学专业: 计算机科学与技术, 当前就职单位于中国移动集 团设计院湖南分院, 职务: 高级咨询设计师, 职称级别: 中级工程师。朱炫滋 (1989-), 女, 毕业于中南大学, 信息 与通信工程专业, 当前就职于中国移动通信集团设计院有限公司湖南分公司, 高级咨询设计师, 中级工程师。周博明 (1986-), 女, 毕业于长沙理工大学, 信息管理与信息系统专业, 当前就职于中国移动通信集团设计院有限公司湖南 分公司, 咨询设计师, 中级工程师。张虹 (1990-), 女, 毕业于湖南师范大学树达学院, 通信工程专业, 当前就职于 中国移动通信集团设计院有限公司湖南分公司, 咨询设计师, 中级工程师。李罡 (1990-), 男, 毕业于湖南师范大学, 通信工程专业, 当前就职于中国移动通信集团设计院有限公司湖南分公司, 咨询设计师, 中级工程师。 